\title{
Alternativas e processos de vigilância em saúde do trabalhador relacionados à exposição ao benzeno no Brasil
}

\author{
Benzene exposures in Brazil: \\ alternatives and processes \\ of worker health surveillance
}

Jorge M esquita H uet M achado 1

Danilo Fernandes Costa 2

Luiza M aria Cardoso 3

Arline Arcuri 3

\footnotetext{
1 Fundação Oswaldo Cruz,

M inistério da Saúde.

Av. Brasil 4.365, Prédio

Quinino, 21045-900,

Rio de Janeiro RJ.

jorgemesquita@yahoo.com.br

2 Departamento de Saúde

e Segurança do Trabalho MTE.

3 Fundacentro MTE.
}

Abstract The article reports the experience of the following up of the Benzene Agreement which is contextualized as a practice of surveillance in the field of Worker's $\mathrm{H}$ ealth. It brings into focus the international facet of that experience as well as the twenty years history of the reduction in the use of benzene in Brazil. The article points at four distinct moments of that history. Firstly, it describes the moment in which the technical bases were built and the several crucial alliances were defined. Then, the shaping of the A greement is described. This process is characterized by conceptual developments, such as the restriction in the use of benzene and the setting up of means of surveillance. Finally, the article relates the results of the process of surveillance, in particular the challenges faced and the proposals to be put into practice in the future. The Brazilian model of surveillance in the field of W orker's $\mathrm{H}$ ealth is debated starting from the understanding of the actions related to the use of benzene and the strengthening of its strategical and structured elements.

Key words Benzene, 0 ccupational health, $\mathrm{H}$ ealth surveillance
Resumo A experiência de acompanhamento do A cordo do Benzeno é relatada e contextualizada como prática de vigilância em saúde do trabalhador. Sendo destacados o processo de senvolvido internacionalmente e a evolução dos últimos 20 anos de história na redução do uso do benzeno no Brasil. A periodização apre sentada aponta para quatro momentos distintos. Inicialmente o momento em que são constituídas as bases técnicas e alianças fundamentais que vão propiciar o acordo; a seguir, o período de formatação do acordo com seus avanços conceituais, de restrição de uso do benzeno e de estabelecimento de formas de acompanhamento; e posteriormente o resultado desse processo de acompanhamento com seus desafios e propostas a serem colocadas em prática no futuro. 0 modelo de vigilância em saúde do trabalhador do Brasil é discutido à luz das ações relacionadas ao benzeno e da consolidação de seus el ementos estratégi cos e estruturantes.

Palavras-chave Benzeno, Saúde do trabalhador, Vigilância em saúde 


\section{H istória internacional de alerta ao benzeno}

As restrições ao uso do benzeno se iniciam ao final do século 19 com a disseminação de processos industriais pela Europa.

Peter Infante (2002), em seu capítulo “Benzene: an historical perspective on American and European occupational setting", inserido no livro Late lessons from early warnings: the precautionary principle 1896-2000, pontifica a evolução do controle da exposição ao benzeno no cenário internacional, especificamente nos EUA.

Os primeiros alertas de efeitos danosos à saúde ocasionados pelo benzeno aparecem no final do século 19, mais precisamente em 1897, com dois episódios relatados nesse mesmo ano, os casos de anemia em mulheres envolvidas na fabricação de pneus de bicicletas na Suécia e um caso de hemorragia em homem jovem empregado em processo de lavagem a seco na França.

Com a 1a Grande Guerra houve um incremento da indústria química e do uso generalizado do benzeno como solvente em vários processos industriais resultando em novos relatos em 1926, ano em que Greenburg publica um artigo com o nome de "Benzol poisoning as an industrial hazard" na revista Public H ealth Repport nos EUA, e em 1928 quando Doloree Borgomano publicam o primeiro caso de leucemia relacionada ao benzeno no Journal de M edicine deLion.

Em 1939 há uma primeira recomendação de substituição do benzeno por parte de investigadores não implementada, no pós-guerra ocorre uma onda de regulamentação do trabaIho, em 1946, apesar de relato de casos de envenenamento por benzeno na faixa de exposição entre 10 e 35ppm, a American Conference of Governmental Industrial H ygienists (ACGIH) recomenda um limite de exposição de 100ppm, que foi progressivamente reduzido para $50 \mathrm{ppm}$ e 35ppm nos anos seguintes.

Ainda em 1948 o American Petroleum Institute (API) conclui que apenas zero de exposição é absolutamente seguro, entretanto recomenda que as indústrias reduzam a exposição para níveis de 50ppm ou menos. Em 1957 novamente reduz-se o valor limite para $25 \mathrm{ppm}$. $\mathrm{N}$ os anos seguintes com esse lapso de prevenção muitos trabalhadores morreram.

A discussão de limites de tolerância se expande, critérios quantitativos de exposição passam a se colocar no centro das medidas de controle. Há a difusão do paradigma de exposição dos riscos físicos para os riscos químicos, esse é o referencial teórico dominante e serve de base na nossa legislação estabelecida na dé cada de 1970, com as normas reguladoras do M inistério do Trabalho e Emprego, bem como fundamento dos critérios para aposentadoria especial adotados pela Previdência Social.

Os primeiros estudos epidemiológicos analíticos são publicados na década de 1970 por Infante et al. (1977) e Aksoy et al. (1974), bases que levam o US Department of Labour em 1977 a tentar reduzir para 1ppm o limite máximo de exposição. Contestado pelo API, o que deu origem a 10 anos de luta judicial, finalmente em 1987 o limite foi estabelecido em $1 \mathrm{ppm}$. Segundo estimativas, tal demora no estabelecimento daquele limite resultou em pelo menos 200 mortes nos EU A. Em 1996 estudos demonstraram ef eitos relacionados com exposições ao benzeno na gasolina a níveis abaixo de 1ppm. I sso fez com que os EUA, em 2001, assumissem a existência de um risco de exposição da população à presença do benzeno no petróleo.

A OM S recomenda para exposição populacional patamares de $3 p p b$, ou seja, valores 330 vezes menores do que o recomendado ocupacionalmente.

Em editorial, a revista Epidemiology (Checkoway, 1993) condena os estudos epidemiológicos como forma de serem estabelecidos patamares de exposição seguras, principalmente pelo tempo de latência entre exposição e efeito crônicos decorrente de exposições químicas. A publicação aponta a toxicologia como mais importante e recomenda a utilização do princípio de precaução como uma política a ser adotada.

Em um estudo de uma coorte de trabalhadores expostos a pelo menos 1ppm/ano, de 10 de janeiro de 1940 a 31 de dezembro de 1976, na indústria de pneus nos EUA, foi demonstrado que a maioria dos casos apresentava de 5 a 10 anos de exposição. A mediana da latência encontrada é de 20 anos com um mínimo de 2 anos e um máximo de 51 anos decorrentes da primeira exposição e o diagnóstico do caso de leucemia (Rinsky, 2002).

\section{A experiência brasileira}

Ao revermos a experiência brasileira de vinte anos de introdução de restrições ao uso do 
benzeno e o controle de seus efeitos no Brasil, destacamos os seguintes períodos de inflexão desse processo que chamamos de vigilância da exposição ao benzeno no Brasil:

1983-1993 - Construindo as Bases

de Intervenção

1994-1995 - Negociação do Acordo

1995-2002 - Acompanhamento do Acordo

2003 - Perspectivas, A ção Integrada

\section{Construindo as bases de intervenção}

Os elementos fundamentais do controle de situações insustentáveis do ponto de vista ambiental ou críticas, como poderíamos chamar a partir da ótica da saúde, serão apresentados com fundamento na experiência de 20 anos de controle da exposição do benzeno no Brasil.

0 benzeno, uma substância reconhecidamente carcinogênica, tem sido objeto de controle no âmbito mundial dada sua característica de contaminante universal e seus potenciais efeitos à saúde humana (Barale, 1995). É considerada a quinta substância de maior risco, segundo os critérios do programa das Nações U nidas de segurança química.

0 marco inicial de seu controle no Brasil é a resolução interministerial de 1983, na qual os ministérios da saúde e do trabalho e emprego, em consonância com representações industriais, estabelecem a redução da contaminação pelo benzeno dos produtos acabados em até $1 \%$ do seu volume, o que representa a primeira iniciativa vitoriosa de redução significativa na exposição do benzeno no Brasil.

Q uase que paral elamente e mesmo com a influência desse debate da exposição ao benzeno, o foco das ações de controle se concentrou na exposição ocupacional com discussão da leucopenia dos trabal hadores siderúrgicos iniciada na Baixada Santista. Em um contexto de redemocratização do estado brasileiro, tais ações passam a permitir alianças do poder público com o movimento sindical, colocando a vigilância epidemiológica e os direitos previdenciários na ordem do dia dos movimentos sociais, ou seja, a luta pela saúde passa a fazer parte da agenda sindical e a saúde dos trabalhadores objeto das políticas públicas.

0 período é também marcante pela discussão da reforma sanitária brasileira sob a influência da italiana, em que a saúde dos trabaIhadores se destaca como um de seus temas centrais (Berlinguer, 1993).
Os anos de 1983-1994 são caracterizados pela construção da estratégia de aliança entre poder público e forças sociais nas lutas por meIhores condições de vida.

A base epidemiológica pode ser estabelecida a partir dos dados consolidados na publicação da Fundacentro (1995) e em estudos pioneiros de Lia Giraldo Augusto (1991;1993) eDanilo Costa (1996).

Os dados sobre morbidade relacionada ao benzeno (Augusto LG 1991; 1993) ea estimativa de casos de leucemia por benzenismo revelam o impacto social potencial dos casos com alterações hematológicas afastados das atividades em situação de exposição a agentes mielotóxicos. Esses números e a base tecnológica existente no setor colocam a siderurgia como prioritária nas ações de vigilância, o que justifica o montante dos gastos em reformas das coquerias, visando à redução da exposição em níveis preconizados pelos instrumentos legais.

A diferença dos valores de referência tecnológicos entre a siderurgia, que é de 2,5ppm, e do setor químico, petroquímico e do petróleo, que é de 1ppm, é fundamentada na dificuldade de se estabelecer um padrão de controle de vazamentos devido a diferenças nas bases tecnológicas entre tais setores. D estaca-se que não há proteção à saúde com esses padrões de controle mesmo com 1ppm segundo estimativas calculadas a partir do modelo da Environmental Protection Agency (U.S.EPA, 1991), apresentadas na tabela 1.

O Estudo de caso Companhia Siderúrgica Paulista (Cosipa), realizado pela Fundacentro e pela Del egacia Regional do Trabalho DRT-SP, em conjunto com o Sindicato de M etalúrgicos da Baixada Santista e o M inistério Público, apresentou na análise da série histórica dos hemogramas dos leucopênicos da Cosipa uma incidência de $46,95 \%$ de alterações hematológicas em cinco anos de acompanhamento de 328 trabalhadores, sendo $15,85 \%$ persistentes (alterações em três ou mais exames), relatório apresentado na CN P-Bz (Costa, 1996).

A tabela 1 apresenta os riscos para cada concentração atmosférica e individual segundo os padrões de exposição.

Considerando que a redução do uso e das exposições fossem decorrentes dos padrões estabelecidos e não ao contrário, como realmente acontece, teríamos uma redução do número de casos ao introduzir os novos padrões do acordo. A redução seria de 630 casos, ou seja, de 720 casos previstos com o padrão de 8ppm pa- 
Tabela 1

Concentração individual de exposição e risco de câncer ocupacional segundo índices de exposição atmosférica ao benzeno.

\begin{tabular}{lcccc}
\hline Indicadores & \multicolumn{3}{c}{ Exposição Atmosférica em ppm } & 1 \\
\hline $\begin{array}{l}\text { Concentração individual } \\
\text { (em mg/m3) }\end{array}$ & 8 & 4 & 2,5 & 0,25 \\
Casos de câncer & 25,60 & 10,07 & 8,00 & \\
Risco & $720^{*}$ & $48^{* *}$ & $30^{* *}$ & $60^{* * *}$ \\
\hline
\end{tabular}

Obs: 8ppm representa o índice permitido na legislação anterior ao acordo; 4ppm representa a média observada na Companhia Siderúrgica Nacional em 1990, e 2,5 e 1ppm são os novos índices propostos como VTR para siderurgia e indústrias de processo respectivamente.

* Considerando 36.000 trabalhadores diretamente expostos

** Considerando 6.000 trabalhadores siderúrgicos expostos diretamente

*** Considerando 30.000 trabalhadores da indústria química, petroquímica e petroleira Fonte: M achado JM H \& M oreno P 1997.

ra 90 casos com os novos padrões da siderurgia e das indústrias químicas, petroquímicas e petroleiras.

Deve-se considerar relativo o impacto da introdução dos VRTs de 2,5 e 1ppm, mas o estabelecimento de novos padrões objetivamente geram novas formas de controles bem como a introdução de novas tecnologias adequadas a esses e a padrões até mais restritos.

\section{Negociação do acordo}

Em 1994 há a negociação do acordo com base no acúmulo do período anterior e a partir da crise estabelecida pela introdução do benzeno como carcinogênico e a proibição de seu uso pelo M inistério de Trabalho.

0 texto acordado, e que supera a crise, éinserido no Anexo 13 NR 15 da lei 3.214 do M inistério do Trabalho e Emprego. São ainda formuladas e publicadas duas instruções normativas, IN 01, que trata da avaliação ambiental, e IN 02, que trata da vigilância em saúde.

\section{Pontos importantes do acordo}

A introdução do Valor de Referência Tecnológico, o VRT, que representa assumir posição de precaução em que não existe exposição segura para o benzeno, supera o conceito de Limites de Tolerância (Castleman \& Ziem, 1988) inadequados para substâncias carcinogênicas como o benzeno, conforme reconhecido pela le- gislação alemã, porém de utilização em larga escala pelas grandes empresas siderúrgicas, petroleiras, químicas e petroquímicas.

A constituição da Comissão N acional Permanente do Benzeno, a CNP-Bz, instância de acompanhamento da aplicação e desenvolvimento do acordo, é estratégica na articulação de ações transversais. A instituição da CN P-Bz, que se configurou em uma estratégia de resistência que vem buscando o aprofundamento do conhecimento da situação epidemiológica e de exposição, tem na disseminação da informação mecanismos de fortalecimento dos movimentos de reestruturação da luta sindical pela saúde dos trabal hadores. Há ainda a mobilização do Estado através da construção de uma rede técnica de difusão de informação em que a criação de regionais de acompanhamento do acordo, a realização de cursos para trabalhadores para formação dos Grupos de Trabalhadores do Benzeno GTBs e o CD do Benzeno são marcos vitoriosos dessa política.

A definição dos GTBs institui a vigilância ao benzeno como uma prioridade de ação para as Comissões Internas de Prevenção de Acidentes Cipas das empresas cadastradas e estabelece concretamente a possibilidade de realização de ações de chão de fábrica, ou seja, de vigilância popular e de exercício do controle social da exposição. Dessa forma a ação de acompanhamento do acordo passa a ser permanente, pois é compartilhada com os trabalhadores no seu cotidiano de trabalho.

0 estabelecimento do Programa de Prevenção da Exposição O cupacional ao Benzeno, o 
PPEOB, consiste em documento técnico de consolidação das ações de controle propostas pelas empresas.

Como forma de restringir o uso e a circulação do benzeno o "Acordo" proíbe o seu uso, admitindo como exceções as empresas que 0 produzem ou o utilizem, em síntese, químicas, siderúrgicas e em laboratórios em situações onde ele não possa ser substituído. Estabelece ainda obrigatoriedade de cadastramento dessas empresas e define as condições por regulamento próprio, cujos pontos principais foram descritos acima.

\section{Resultados da ação da CN P-Benzeno}

As vertentes do acompanhamento técnico do acordo nacional do benzeno e suas formas de integração aprofundam a reflexão sobre as ações de vigilância em saúde dos trabalhadores em geral, e constroem uma forma de agir institucional transversal.

A diversidade e a intensidade desse acompanhamento podem ser deduzidas a partir de seus pontos concretos de interven ção, que passamos a descrever.

1. A proibição do uso do benzeno na produção de álcool anidro evitou a exposição de miIhares de trabalhadores a este agente, assim como a circulação de centenas de caminhões trans portadores pelas estradas.

2. 0 estabelecimento de indicador biológico de exposição ao benzeno em substituição ao fenol urinário adequou o indicador de exposição aos índices das concentrações atmosféricas acordados nos Val ores de Referência Tecnológicos, ou seja, exposições abaixo de 2,5ppms. Nesse contexto 0 ácido trans-trans mucônico é considerado eficiente e recomendado.

3. A publicação de nota técnica explicitou que as plataformas e terminais estivessem incluídos no acordo do benzeno. Definiu tais operações como áreas de risco, dada a manipulação de naftas com teores acima de $1 \%$ de benzeno; apontou para que operações que envolvam naftas com menos de $1 \%$ também sejam consideradas de risco, devendo ser objeto de controle similar.

4. A definição de um cronograma para diminuição da porcentagem de benzeno em produto acabado reduziu a circulação do benzeno em situações de trabal ho que utilizam produtos como tiners e solventes de borracha e também sua circulação ambiental em geral com a restrição de uso nos produtos domissanitários de maneira imediata pela Anvisa para no máximo uma contaminação de $0,1 \%$ de benzeno e a rotulagem de produtos anunciando a presença de 0,01 a 0,1\% de contaminação por benzeno.

5. A formulação e a aprovação da N orma de Vigilância da Saúde dos Trabalhadores Expostos ao Benzeno, para acompanhamento e diagnóstico dos casos definiram procedimentos padronizados acordados tecnicamente por hematologistas, assessores internacionais e representantes institucionais do governo.

6. A aprovação e adoção do SIM PEAQ (Sistema de M onitoramento de Populações Expostas a Agentes Químicos) permitirão o acompanhamento a médio e longo prazo dos trabalhadores que trabalharam, trabal ham e dos que virão a trabalhar nas empresas que manipulam 0 benzeno, construindo a possibilidade de acoIhimento institucional desses trabal hadorese uma aproximação da real dimensão dos efeitos decorrentes da exposição ao benzeno no Brasil. 7. A realização de visitas técnicas às cinco siderurgias integradas que utilizam gás de coqueria e às quatro empresas produtoras de benzeno permitiu um olhar mais próximo e crítico de como as empresas estavam aplicando as diretivas do acordo. Inicialmente, resultou em uma crise com o setor siderúrgico que, ao se superar, possibilitou um outro ciclo de negociações de novos itens como procedimentos recomendáveis e o aprimoramento de itens como o GTB e os V alores de Referência Tecnológicos, bem como abriu a possibilidade de participação das empresas nas comissões regionais.

8. A Organização de diversos cursos, palestras, seminários, inclusive um internacional consolidou a experiência nacional e propiciou a difusão de informações e a formação de novos técnicos e trabal hadores, que se tornaram aptos para o acompanhamento do acordo no nível institucional, empresarial e das representações dos trabalhadores seja através dos GTBs ou sindicais nas comissões regionais e nacional. A produção do CD-Rom Repertório brasileiro do benzeno ( 1 a edição em 2001 e a 2a em 2002) é um exemplo simbólico.

9. A apresentação de trabalhos em congressos e publicações em revistas nacionais e internacionais consolidou e aprofundou, do ponto de vista acadêmico, a experiência política e institucional do acompanhamento do acordo.

10. A organização de comissões regionais para acompanhamento do acordo do benzeno fez 
com que 0 acompanhamento fosse multiplicado, estabelecendo uma rede de controle.

11. A realização de oficina para discussão das condições de trabalho das siderúrgicas e estabelecimento de propostas delineou um novo momento de relacionamento com o setor, bem como evidenciou o investimento das empresas siderúrgicas em controle de vazamentos, porém com menos eficiência do que as petroquímicas.

12. A realização de oficina para discussão das condições de trabal ho das petroquímicas e estabelecimento e propostas apontou para uma general ização ao setor petroleiro do investimento das empresas petroquímicas em controle coletivo.

13. A publicação de Portaria do Geisat recomendando a criação de núcleos de diagnóstico de benzenismo deve consolidar a integração institucional para acompanhamento e diagnóstico dos casos de benzenismo.

14. 0 estabel ecimento de critérios de retorno para os trabalhadores com alta do INSS permite um retorno com acompanhamento institucional e pelos representantes regionais dos trabalhadores e estabelece critérios de avaliações epidemiológica, ambiental e técnica.

15. A influência na portaria da Agência N acional do Petróleo (ANP) sobre o teor de benzeno em gasolinas fez valer a portaria de 1983 como critério de contaminação máxima para gasolina comum, reduzindo a exposição ambiental e fomentando projetos internos nas refinarias para redução dos teores de benzeno nas linhas de naftas, reduzindo assim também a exposição ocupacional nas refinarias.

\section{Desafios}

Os desafios definidos em 2003 são descritos a seguir:

1. Fomentar intervenções tecnológicas para re dução de exposição, principal mente no que se refere à utilização do gás de coqueria no setor siderúrgico, por meio do combate aos vazamentos e de sua substituição como fonte de calor utilizada em fornos, que por sua vez necessitam de quilômetros de tubulações para distribuição do gás no interior dos parques industriais.

2. Iniciar o processo de estabelecimento da sustentabilidade do uso do petróleo como base energética, definindo como meta a redução progressiva do impacto ambiental da produção e uso do petróleo. Discussão da tecnologia das refinarias, dos terminais e de transporte de naftas com a revisão dos controles de vazamentos e dos acidentes; estabelecer indicadores de conformidade.

3. Revisar o silêncio epidemiológico, implantar centros de referência para diagnóstico e estabelecer critérios de diagnóstico padronizado e um sistema de informações para acompanhamento dos casos e dos grupos envolvidos em atividades onde se concentrem os programas de redução de exposição.

4. Enfrentar a discriminação hematológica, promover campanha de esclarecimento aos médicos que estão envolvidos em processos de demissão e admissão das empresas para adotarem critérios éticos recomendados na $\mathrm{N}$ orma de $\mathrm{Vi}$ gilância da Exposição ao Benzeno no Brasil.

5. Ampliar o foco das ações de vigilância abrangendo o controle do benzeno na gasolina, principalmente estabelecendo programas de redução da exposição aos frentistas e mecânicos que manipulam diretamente combustíveis. 6. Realizar a revisão do acordo, cabendo à bancada do governo a apresentação das melhores práticas para reduzir a exposição ao benzeno. À bancada dos trabal hadores cabe apresentar propostas de avan ços na regulação dos GTBs, ficando a bancada dos empregadores encarregada de apresentar proposta de redução do VRT. 7. Quanto à integração interinstitucional, 0 momento atual é de redefinição no sentido de uma melhor articulação das ações de governo, principalmente por parte da Previdência Social revendo seu papel na identificação e registro de casos de doenças relacionadas ao trabalho. Passando a associar-se ao SUS e às DRTs no processo de busca ativa de casos de benzenismo, assumindo a análise sistemática a partir dos procedimentos apontados na Norma de Vigilância da Saúde dos Trabal hadores Expostos ao Benzeno no Brasil do M inistério da Saúde.

\section{Considerações finais}

M esmo sob fortes tensões, o acúmulo proveniente da interação fez com que o modelo de vigilância da exposição ao benzeno fosse ficando cada vez mais complexo e claro, reafirmando a necessidade de uma abordagem múltipla e transversal. Para construirmos um modelo de vigilância podemos aplicar o esquema desenvolvido por M achado (1996), no qual a Vigilância em Saúde do Trabal hador integra componentes da atividade, da qualidade do risco e 
do tipo de efeito em um determinado espaço em uma perspectiva transdisciplinar em que são integradas múltiplas formas de acompanhamento com suas distintas bases disciplinares (Figura 1).

Nesse caso, o ponto de partida foi a intervenção inicial sobre uma situação de risco, com a redução do benzeno em produtos acabados, passando posteriormente para identificação de casos relacionados à exposi ção na atividade siderúrgica. Posteriormente, há um deslocamento redefinindo o foco de ação da vigilância para o benzeno, um tipo de risco específico, em torno do qual se consolidam as ações de vigilância.

Podemos sintetizar como campos de vigilância consolidados pela experiência do benzeno no Brasil:

1. Vigilância tecnológica de redução e eliminação dos riscos.

Com a implantação de alternativas tecnológicas de substituição, enclausuramento e redução da emissão atmosférica do benzeno e eliminação do benzeno em diversos produtos. Estabelecimento de procedimentos, recomendados nas atividades de carregamento, laboratoriais, principalmente no processo de lavagem; procedimentos de coleta de amostras e drenagens de linha, projetos de redução de emissão nos separadores água-óleo, a substituição de equipamentos mais adequados e de procedimentos de manutenção em linhas e equipamentos envolvidos nos processos com a presença de benzeno.

2. Vigilância epidemiológica de avaliação dos efeitos e formas de exposição.

Com a organização das informações relativas aos casos antigos, à série histórica dos trabalhadores expostos a situações de risco, à adequação dos serviços de saúde das empresas, do SUS e perícia do INSS, no sentido de identificar e acompanhar os casos de benzenismo.

Com a implantação do monitoramento da morbimortalidade dos trabal hadores expostos ao benzeno, estabelecendo a incidência de benzenismo e de casos suspeitos na população ocupacionalmente exposta.

Com o estabelecimento de fluxo de informações entre as empresas e os departamentos de dados vitais do SU S com a implantação do SIM PEAQ.

3. Vigilância ambiental de monitoramento da exposição, incorporando metodologias participativas e de higiene industrial.

Com a realização sistemática de ações de vigilância nos locais de trabal ho com a parti- cipação dos trabal hadores (O ddone,1986). Como símbolos dessa vertente estão a discussão dos Grupos de Trabalhadores para o Benzeno, os GTBs, organizados no interior das empresas, e o controle das emissões atmosféricas de benzeno, com o estabelecimento de limites tecnológicos de exposição, índices técnicos possíveis de serem alcançados, considerando a base tecnológica do momento em cada setor produtivo.

As atividades de acompanhamento nos locais de trabalho do acordo nacional do benzeno carecem de uma definição de metodologia de interpretação dos resultados das avaliações ambientais, de formas de revisão de amostragens, de validação laboratorial e estabel ecimento de programas de controle de qualidade interlaboratoriais através da organização de redes de referência, bem como a revisão metodológica e de identificação de vieses de seleção de grupos homogêneos de exposição.

Nesse sentido, está prevista uma oficina técnica para o segundo semestre de 2003 na Fundacentro (São Paulo) para discussão da IN 02 que define as formas de avaliação ambiental no acordo, que, com a revisão do VRT pelas empresas, consolidam um processo de análise das práticas em execução e com a perspectiva de padronização adequada.

Considera-se ainda que deve ser retirado do centro do imaginário dos serviços das empresas a idéia de limites de tolerância, conceito funesto para saúde dos trabalhadores. Esses limites não levam em consideração variações de absorção de sensibilidade e mesmo ambientais, são apenas parâmetros de evolução de controle técnico.

\section{Figura 1}

M atriz de ação em vigilância em saúde do trabalhador.

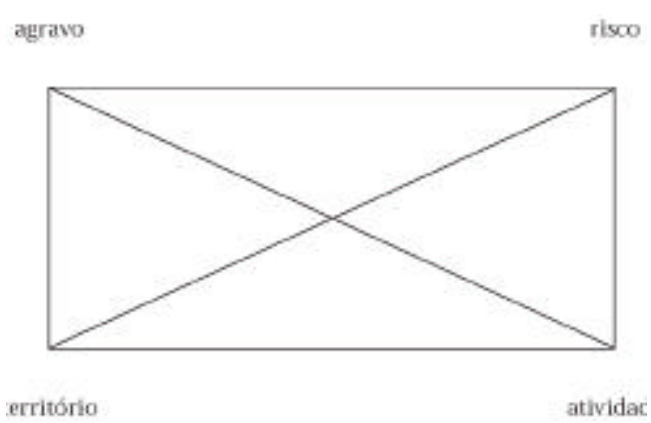

Fonte: M achado, 1996 


\section{Perspectivas para construção de um modelo de vigilância em saúde do trabalhador no Brasil}

Algumas lições aprendidas com essa experiência de construção de um modelo de vigilância para o benzeno no Brasil podem ser generalizadas para que sejam definidos um ou vários modelos a partir de outros casos com recortes e objetos distintos.

0 modelo de vigilância aqui apresentado parte da situação de risco ou do agravo ou mesmo da conjunção de ambas as situações (Baker, 1989) e se fortalece quando integrado à noção de território, em que diversos atores e instâncias institucionais desempenham suas ações configurando um campo de forças de vigilância que vai atingir o outro elemento fundamental do modelo, o processo de trabalho, representado pela atividade e pelo setor onde se concentram os casos e as exposições, representado na figura 1.

A matriz de vigilância é desigual e se comporta dinamicamente em um processo que parte do campo mais tradicional do paradigma existente e dominante, tanto do ponto de vista da saúde como do controle dos riscos exercido pelas empresas em seu próprio ambiente.

Ao introduzir o tema de vigilância da saúde do trabalhador em seus serviços, o setor da saúde traz como proposição, como objeto da vigilância, um indicador de efeito, no nosso caso a leucopenia (Augusto \& N ovaes, 1999), marcando o modelo pela influência da vigilância epidemiológica. Pelo lado do controle do ambien-

\section{Figura 2}

M atriz de vigilância em saúde do trabalhador com a direcionalidade do processo de construção de sua capacidade de resistência.

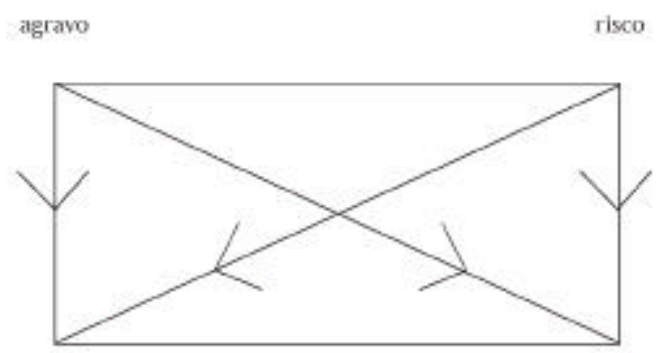

território

atividade te de trabalho é proposto que sejam estabelecidos parâmetros de redução da exposição ao benzeno, fortalecendo o modelo das empresas, que têm como paradigma central a vigilância de agentes de risco, com metodologias centradas na Higiene Industrial e nos parâmetros quantitativos de exposição.

0 desafio e o fator de diferenciação do modelo é a construção de uma direcionalidade da matriz de vigilância no sentido do aprofundamento da relação dos agravos e riscos com 0 território e a atividade, conforme figura 2 .

Ao aprofundarmos a relação com a atividade estamos nos aproximando da relação processo de trabalho e saúde, objeto central do campo da saúde do trabal hador (Laurell \& Noriega, 1989).

Por sua vez, o território representa a dinâmica social dada pela participação dos trabalhadores e pela construção social do espaço ( $M$ endes, 1994), elemento fundamental na construção de redes de resistência do modelo, para que este avance nos seus propósitos de promoção da saúde e elemento analítico do contexto das ações e das bases sociotécnicas inseridas e condicionantes da relação da saúde com o objeto da ação de vigilância.

Portanto, o modelo construído com a ênfase nos pólos do território e atividade agl utina o contexto social etécnico ampliando o processo de vigilância para além da relação com os pólos tradicionais de vigilância ambiental, ou seja os riscos e a vigilância epidemiológica, os agravos, de forma a tornar mais complexo o modelo tornando-o histórico e construtivista, Em uma perspectiva técnica que aumenta sua resistência e potencializa sua intervenção, o processo de vigilância alcança um status de processo político intelectual onde são criadas redes de sustentação sociais amplas e de intervenção cada vez mais abrangentes.

Tanto o risco como o efeito são conseqüências das condições de trabalho, das tecnologias aplicadas e da forma em que os trabalhadores objetivamente exercem suas atividades. Q ualquer ação de vigilância que não leve em consideração essa simples questão tende a ser incompleta. 


\section{Referências bibliográficas}

Aksoy M , Erdem S \& Dincol G 1974. Leukemia in shoeworkers exposed chronically to benzene. Blood 44: 837-841.

Augusto LG 1991. Estudo longitudinal e morfológico (medula óssea) em pacientes com neutropenia secundária à exposição ocupacional crônica ao benzeno. Dissertação de mestrado. Universidade de Campinas, Campinas.

Augusto LG 1993. Histological damage in bone marrow by exposure to benzene and follow up to peripherical blood count of workers with chronic intoxication. Revista Brasileira de Saúde 0 cupacional 21(78): 86-92.

Augusto LG \& N ovaes TC 1999. Ação médico-social no caso do benzenismo em Cubatão, São Paulo: uma experiência de interdisciplinaridade. Cadernos de Saúde Pública 15(4):729-738.

Baker EL 1989. Sentinel Event N otification System for O ccupational Risks (SENSOR): the concept. American Journal of Public H ealth 79 (supplement): $18-20$

Barale R 1995. Genotossità del benzene, pp. 41-50. In M inoia C, A postoli P\& Bartolucci GB (orgs.). II benzene: tossicologia, ambienti di vita edi lavoro. M organ Ed., Milão.

Berlinguer G 1993. A saúde nas fábricas. Hucitec, São Paulo.

Castleman BI \& Ziem GE 1988. Corporate influence on threshold limits values. American Journal of Industrial M edicine 13:531-559.

Checkoway H 1993. Dertermining the hazards of workplace chemicals. Epidemiology 4:91-92.

Costa DF et al 1989. Programa de saúde dos trabalhadores, a experiência da Zona N orte: uma alternativa em saúde pública. Hucitec, São Paulo.

Costa D 1996. Relatório da análise de hemogramas dos trabalhadores da Coqueria e Carboquímicos da Cosipa. M inistério do Trabalho, São Paulo.
Fundacentro (Fundação Jorde Duprat Figueiredo de Segurança e M edicina do Trabalho) 1995. Benzeno. São Paulo.

Health \& Safety Executive 1990. Surveillance of people exposed to health risks at work. HS(G) series, Londres.

Infante PF et al. 1977a. Leukemia in nenzene workers. Lancet 2:76-78.

Infante PF et al. 1977b. Benzene and leukemia. Lancet 2: 217-245.

Infante PF 2002. Benzene: an historical perspective on American and European occupational setting. In Late lessons fom early warnings: the precautionary principle 1896-2000.

Laurell AC \& Noriega M 1989. Processo de produção e saúde: trabalho e desgaste operário. Ed. Hucitec, São Paulo.

M achado JM H 1996. A heterogeneidade da intervenção: alternativas de vigilância em saúde do trabalhador. Tese de doutorado. Escola N acional de Saúde Pública da Fiocruz, Rio de Janeiro.

M achado JM H. \& M oreno P 1997. M apeamento das áreas industriais que utilizam e produzem benzeno no Brasil. Fundação Oswaldo Cruz, Rio de Janeiro. (M imeo).

M endes EV 1994. $O$ distrito sanitário. Hucitec, São Paulo.

Oddone I et al. 1986. 0 ambiente de trabalho: a luta dos trabalhadores pela saúde. Hucitec, São Paulo.

Rinsky RA, H ornung RW, Silver SR \& Tseng CY 2002. Benzene exposure and hematopoietic mortality: a long-term epidemiologic risk assessment. American Journal of Industrial M edicine 42:474-480.

US EPA, Office of Solid Waste and Emergency Response 1991. Risk Assessment Guidance for Superfund, Volume 1: Human H ealth Evaluation M anual Supplemental Guidance: Standard D efault Exposure Factors. Directive 9285.6-03; Interim Final. M arch 25.

Artigo apresentado em 28/8/2003

Aprovado em 20/10/2003

Versão final apresentada em 4/11/2003 